line $\lambda 3471 \mathrm{~A}$. has been produced in the laboratory. Further, while the dissociation during the day by absorption of ultra-violet light from the sun is certainly very slight, any other process seemed impossible ; we thought that the energy for excitation of the light of the night sky-which does not exceed 7 electron volts-is not sufficient to dissociate the nitrogen molecule. But this energy of dissociation was not accurately known; the value $(9 \cdot 0$ volts) accepted a few years ago is now recognized as too high; the value adopted to-day ${ }^{5}$ is 7.34 volts. We can consequently accept the dissociation of nitrogen molecules.

In a recent paper, Bernard ${ }^{6}$ shows that a radiation at $\lambda 3471 \mathrm{~A}$. is also present in the spectrum of diffuse auroras, where the Vegard-Kaplan bands are particularly intense. This is an interesting confirmation of the probable presence of metastable $N_{I}$ atoms in the high atmosphere. But Bernard's paper requires two comments :

(1) Vegard and his collaborators probably observed the line $\lambda 3471 \mathrm{~A}$. in the aurora; they did not separate it from the second-positive band near $3469 \mathrm{~A}$.; the intensity attributed to this band is evidently too high.

(2) The line $\lambda 5206 \mathrm{~A}$., which corresponds to the ${ }^{2} D-{ }^{4} S$ transition of $N I$, has not yet been recognized in the night sky; but its existence in the spectrum of the aurora was pointed out by V. M. Slipher and L. A. Sommer ${ }^{7}$, who suggested its origin and mentioned that the line had been observed ${ }^{8}$.

In conclusion, I propose seeking the $\lambda 5206 \mathrm{~A}$. line in the spectrum of the night sky by a right choice of photographic plates and studying the $\lambda .3471 \mathrm{~A}$. radiation of the night sky by the interferometer method, in order to ascertain for certain the existence of nitrogen atoms in the high atmosphere.

Observatoire de Lyon.

J. GaUZit.

Aug. 2.

${ }^{1}$ Kaplan, NATURE, 141, 645 and 1139 (1938).

${ }^{2}$ Dufay, J. Phys., vii, 5, 523 (1934).

Gauzit, J. Phys., vii, 5, 527 (1934).

- Kaplan, Quatrième rapport de la Commission pour l'étude de relations entre les phénomènes solaires et terrestres, p. 140 (1936)

- See, for example, Mathur, L. S., and Sen-Gupta, P. K., Proc. Acad. Sei. U.P., 5, Part 2, 187-226 (1938).

- Bernard, NATURe, 141, 1141 (1938).

' Slipher and Sommer, Naturwiss., 171, 802 (1929):

- See Kayser, "Handbuch der Spektroscopie", 5, 57

\section{Angular Distribution of Electron Pairs}

A NUMBER of experiments have been made on the angular distribution of electron-positron pairs liberated by a beam of $\gamma$-rays, but the results, where it is possible to compare them, do not seem to be in agreement. It is therefore of interest to see what the theory predicts, and in the following a short account is given of the results obtained by a rigorous treatment of the problem. The method used is an extension of that given by $\mathrm{us}^{1}$ to find the total number of pairs created. So far, the calculations have been made for $Z$ (atomic number) $=84$, $h \nu=5 m c^{2}$, and $Z=50, h \nu=3 m c^{2}$ and $5 m c^{2}$, where $h \nu$ is the energy of the $\gamma$-ray.

Denoting the average angles made by the direction of the electron and positron with the direction of the $\gamma$-ray by $\theta_{e}$ and $\theta_{p}$ respectively, and the average angle between the pair by $\gamma$, the following results were obtained.
(1) $\theta_{e}, \theta_{p}$ and $\gamma$ decrease with increasing energy of the $\gamma$-ray. For particles of definite energy, the formula given by Bethe and Heitler ${ }^{2}$ for the number of electrons of energy $E_{0}$ emitted at an angle $\theta_{0}$

$$
\Phi\left(\theta_{0}\right) d \theta_{0}=\frac{\theta_{0} d \theta_{0}}{\left(\Theta^{2}+\theta_{0}{ }^{2}\right)^{2}}, \Theta=\frac{m c^{2}}{E_{0}},
$$

is not valid for such low energies. The values found for $\theta_{e}, \theta_{p}$ and $\gamma$ when $Z=84$ are about twice those given by the above formula $\left(h \nu=5 m c^{2}\right)$.

(2) $\theta_{e}$ is slightly greater than $\theta_{p}$. This is to be expected since the average energy of the positron is greater than that of the electron. This is in qualitative agreement with Grosev and Frank ${ }^{3}$, who find for krypton $\theta_{e}=33^{\circ}, \theta_{p}=30^{\circ}$.

(3) $\theta_{e}, \theta_{p}$ and $\gamma$ increase slowly with $Z$. It is easy to show that the angular distribution is independent of $Z$ to the first order (Born approximation), but for large $Z$ the electrostatic forces on the particles become important, and these must be responsible for the increased values of $\theta_{e}, \theta_{p}$ and $\gamma$ noted in (1). The strongest evidence in support of this would seem to be Alichanian's experiments ${ }^{4}$ on lead, with $h \nu=14 m c^{2}$. $\mathrm{He}$ found $\gamma$ to be about $65^{\circ}$, as compared with $48^{\circ}$ in krypton $^{3}$ for a considerably lower energy $(h \nu=$ $5 m c^{2}$ ). Similar results have been obtained by Jelepov (ref. 3, p. 52, footnote).

The calculations involved in the use of the accurate wave functions are extremely laborious, particularly the evaluation of $\gamma$, the angle between the pair. For purposes of comparison with the theory it is therefore advantageous to have experimental results for $\theta_{e}$ and $\theta_{p}$. It is hoped to publish fuller details and results shortly.

\section{University of Tasmania.}

$$
\text { J. C. JAEGer. }
$$

Royal Observatory, Greenwich.

1 Jaeger and Hulme, Proc. Roy. Soc., A, 153, 443 (1936).

2 Bethe and Heitler, Proc. Roy. Soc., A, 146, 83 (1934).

${ }^{3}$ Grosev and Frank, Comptes rendus (Doklady), 19, 52 (1938).

- Alichanian, Dzelepov and Spivac, Comptes rendus (Doklady), 19, 379 (1938).

\section{Chemical Nature of Proliferation-Promoting Factors from Injured Cells}

WE have published investigations indicating that yeast ${ }^{1}$ and animal cells ${ }^{2}$ injured by ultra-violet light and other means produce factors ('intercellular wound hormones' $)^{3}$ which are apparently released by injured, but living, cells into the intercellular fluid and stimulate the proliferation of normal cells. Spectrographic and chemical studies indicate that the active materials from yeast contain nucleic acid derivatives, as previously suggested ${ }^{4}$.

The 'wound hormones' were produced by prolonged, low-intensity, full ultra-violet irradiation of yeast in isotonic salt solution or distilled water. Cellfree Berkefeld filtrates from irradiated and nonirradiated suspensions were assayed in yeast growth experiments. The biological potency and extinction at $2600 \mathrm{~A}$. were much more marked in the filtrates from irradiated suspensions, and the potency and $2600 \mathrm{~A}$. extinction in various samples were closely parallel.

As shown on the accompanying graphs, the spectrum of the 'wound hormone', or factor from injured cells (obtained by photographing the spectrum 